\title{
Time-Resolved Raman Spectroscopy of Polaron Formation in a Polymer Photocatalyst
}

\author{
Verity L. Piercy, Khezar H. Saeed, Andrew W. Prentice, Gaia Neri, Chao Li, Adrian M. Gardner, \\ Yang Bai, Reiner Sebastian Sprick, Igor V. Sazanovich, Andrew I. Cooper, Matthew J. Rosseinsky, \\ Martijn A. Zwijnenburg,* and Alexander J. Cowan*
}

Cite This: J. Phys. Chem. Lett. 2021, 12, 10899-10905

Read Online

\section{ACCESS | Llll Metrics \& More | 回 Article Recommendations | (I) Supporting Information}

ABSTRACT: Polymer photocatalysts are a synthetically diverse class of materials that can be used for the production of solar fuels such as $\mathrm{H}_{2}$, but the underlying mechanisms by which they operate are poorly understood. Time-resolved vibrational spectroscopy provides a powerful structure-specific probe of photogenerated species. Here we report the use of time-resolved resonance Raman $\left(\mathrm{TR}^{3}\right)$ spectroscopy to study the formation of polaron pairs and electron polarons in one of the most active linear polymer photocatalysts for $\mathrm{H}_{2}$ production, poly(dibenzo$[b, d]$ thiophene sulfone), P10. We identify that polaron-pair formation prior to thermalization of the initially generated excited states is an important pathway for the generation of long-lived photoelectrons.

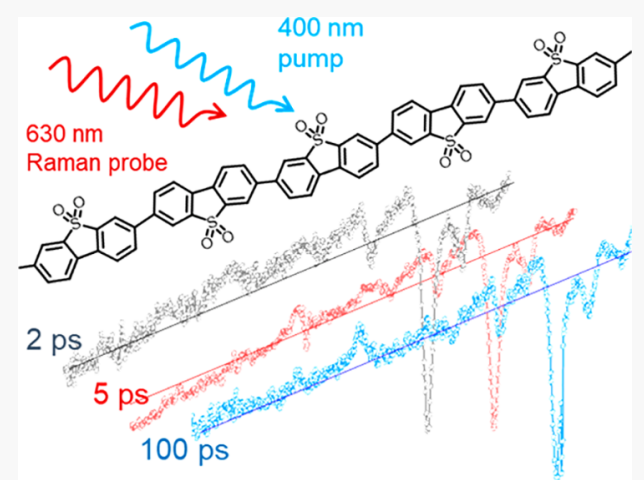

$\mathrm{T}$ he development of scalable photocatalysts that can split water efficiently by using solar energy would transform the energy landscape, providing a way to generate hydrogen sustainably. Historically, research has focused on inorganic semiconductors, but in the past 12 years there has been a rapid increase in the study of organic photocatalysts for water splitting $^{1}$ following studies in 2009 which showed that graphitic carbon nitride was an effective hydrogen evolution photocatalyst. $^{2}$ More recently, a wider variety of classes of organic photocatalysts have been reported including polymeric networks such as conjugated microporous polymers (CMPs), ${ }^{3}$ covalent triazine-based frameworks (CTFs), ${ }^{4-6}$ covalent organic frameworks (COFs), ${ }^{7-9}$ and linear conjugated polymers. ${ }^{10,11}$ Among these, the linear homopolymer of dibenzo $[b, d]$ thiophene sulfone (P10, Scheme 1) was shown to be one of the most active for hydrogen evolution, both when using a sacrificial electron donor ${ }^{12}$ and in a z-scheme water splitting system. ${ }^{13} \mathrm{P} 10$ can also promote oxygen evolution ${ }^{14}$ and $\mathrm{CO}_{2}$ reduction, ${ }^{15}$ all under visible light irradiation.

To facilitate the design of polymer photocatalysts and to truly exploit the synthetic control available, it is important to understand their underlying photophysics and mechanisms. In contrast to inorganic semiconductors, where the photogeneration of free charges occurs with a high efficiency, the poor dielectric screening of charges in organic absorbers means that polaron yields are often low; this is a central issue for this class of materials. Understanding why particular organic photocatalysts can efficiently generate separated charges following photon absorption is important. A body of literature exists on the underlying mechanisms of ultrafast polaron formation in organic photovoltaic (OPV) materials ${ }^{16-19}$ with proposed mechanisms including formation via hot and relaxed exciton dissociation and direct polaron-pair photogeneration. However, it is not clear if such models are also directly applicable to polymer photocatalysts where the additional presence of metal catalysts (e.g., for $\mathrm{H}_{2}$ and $\mathrm{O}_{2}$ evolution) and water may play an important role.

Transient absorption (TA) UV-vis spectroscopy is an established technique that has been widely applied to study electron-hole dynamics of inorganic and organic solar fuel materials. $^{20,21}$ TA studies of $\mathrm{P} 10^{12-14,22,23}$ report initial formation of a broad positive absorption at $>700 \mathrm{~nm}$ assigned to a singlet excitonic state that decays on the picosecond time scale. $^{12}$ In the presence of a sacrificial electron donor (commonly triethylamine (TEA) in a methanol/water solvent, 1:1:1 vol), a long-lived band at $630 \mathrm{~nm}$ has been assigned to an electron polaron $\left(\mathrm{P} 10\left(\mathrm{e}^{-}\right)\right.$), proposed to form by quenching of the excitonic state by TEA on a time scale between 1 and 100 ps. $^{23,12}$ In the absence of a sacrificial electron donor, a $630 \mathrm{~nm}$ TA band is still observed, and this has been proposed to be due

Received: September 17, 2021

Accepted: October 28, 2021

Published: November 3, 2021 
Scheme 1. Kerr-Gated Time-Resolved Resonant Raman Experiment of P10 (Structure Upper Left) ${ }^{a}$

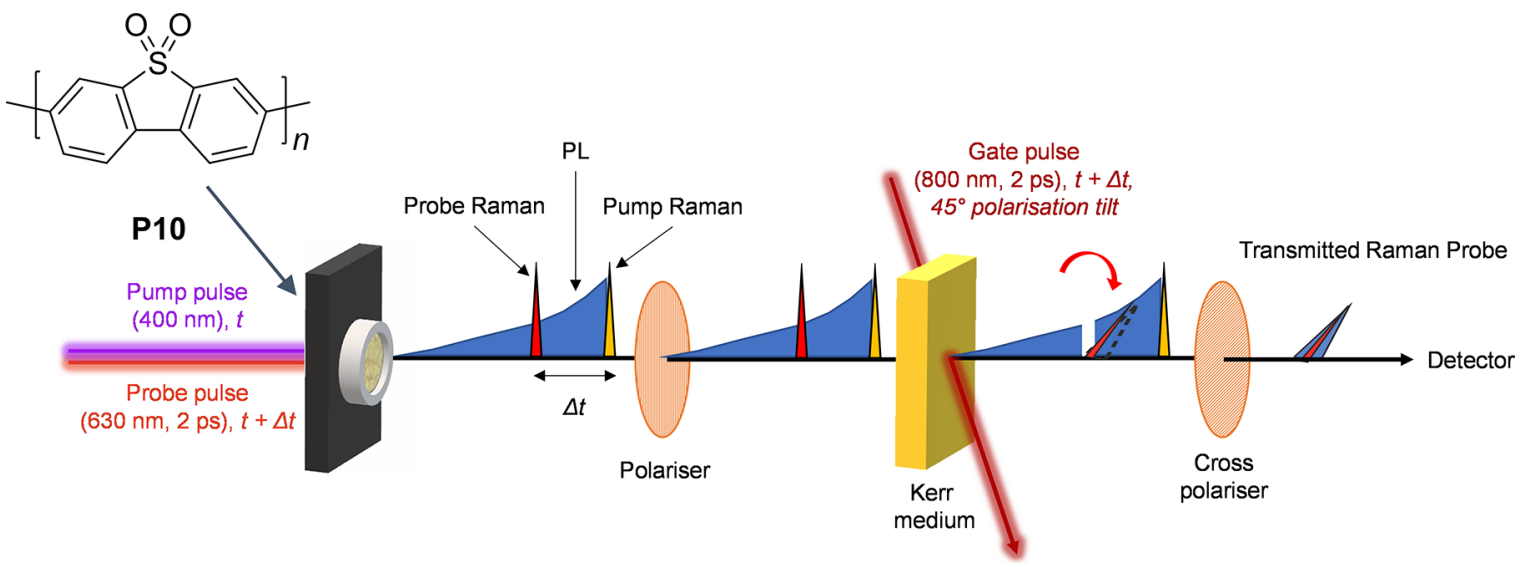

${ }^{a_{T}}$ The laser pump pulse $(400 \mathrm{~nm})$ generates photoexcited states that can be subsequently interrogated by the probe pulse $(630 \mathrm{~nm})$ which is delayed with respect to the pump $(\Delta t)$. The wavelength of this probe pulse was selected to be resonant with an electronic transition associated with a transient species observed in the transient absorption spectra of P10. The probe pulse generates both Raman scatter and photoluminescence (PL). An $\sim 2$ ps duration $800 \mathrm{~nm}$ high-energy laser pulse induced a transient optical anisotropy which lasts for approximately the duration of the gating pulse in the $\mathrm{CS}_{2}$ Kerr medium. During this period the gate is "opened", and the polarization of the incident linearly polarized light is rotated by $90^{\circ}$ with respect to its original orientation and passed through the crossed exit polarizer into the spectrometer while the unrotated light is rejected. Raman scattering is a fast process that occurs on a subpicosecond time scale. By synchronizing the timing of the Raman probe laser pulse with the gate pulse, it is possible to selectively transmit Raman scattered photons while rejecting the majority of the much longer lived (ns or greater) PL.

to a polaron pair, a spatially separated weakly interacting electron and hole, with similar spectral characteristic to the $\mathrm{P} 10\left(\mathrm{e}^{-}\right) .^{12,13}$ The $\mathrm{P} 10\left(\mathrm{e}^{-}\right)$is very stable as it is retained on the polymer chain for $\sim 100 \mu \mathrm{s}$, despite the presence of residual Pd in the structure from the polymer synthesis which acts as a hydrogen evolution catalyst. ${ }^{23}$ For P10, fast polaron formation and trapping of the electron on the polymer leads to a high level of photocatalytic activity, but the chemical nature and mechanism of polaron formation are unclear.

Interpretation and assignment of TA features can be challenging due to the number of broad, often overlapped $\mathrm{UV}-$ vis bands. Time-resolved resonance Raman $\left(\mathrm{TR}^{3}\right)$ spectroscopy directly probes the vibrational modes of shortlived intermediates, enabling assignment of nonequilibrium structures. Raman modes are sensitive to both the local structure and the intermolecular ordering of polymers, making $\mathrm{TR}^{3}$ a potentially useful way to study the mechanism and site of polaron formation. ${ }^{24}$ Time-resolved Raman spectroscopy has been used to study exciton conformational changes and polaron formation in OPV materials but has not been previously applied to study polymer photocatalysts. ${ }^{24-27}$ Here we apply $\mathrm{TR}^{3}$ to study the mechanisms of $\mathrm{P} 10$ polaron formation.

The ground state Raman spectrum $\left(600-1400 \mathrm{~cm}^{-1}\right)$ of P10 powder (633 nm Raman probe) shows peaks at 1411, 1340, 1301, 1269, and $1145 \mathrm{~cm}^{-1}$ (Figure 1a) and a strong band at $1596 \mathrm{~cm}^{-1}$ (Figure S1) that is outside the spectral window used for the $\mathrm{TR}^{3}$ experiment. These bands are assigned to ring/carbon backbone modes except for 1145 $\mathrm{cm}^{-1}$, which has contributions from the sulfone mode through comparison to Raman spectra predicted by density functional theory (DFT) calculations (Supporting Information, Figure S2 and Table S1); see section 1.4 of the Supporting Information for details about the computational method employed. TA experiments performed on P10 aggregates, formed from a toluene suspension, are shown in Figure S4. Toluene is used as an inert, nonpolar solvent to generate a thin layer of P10 for

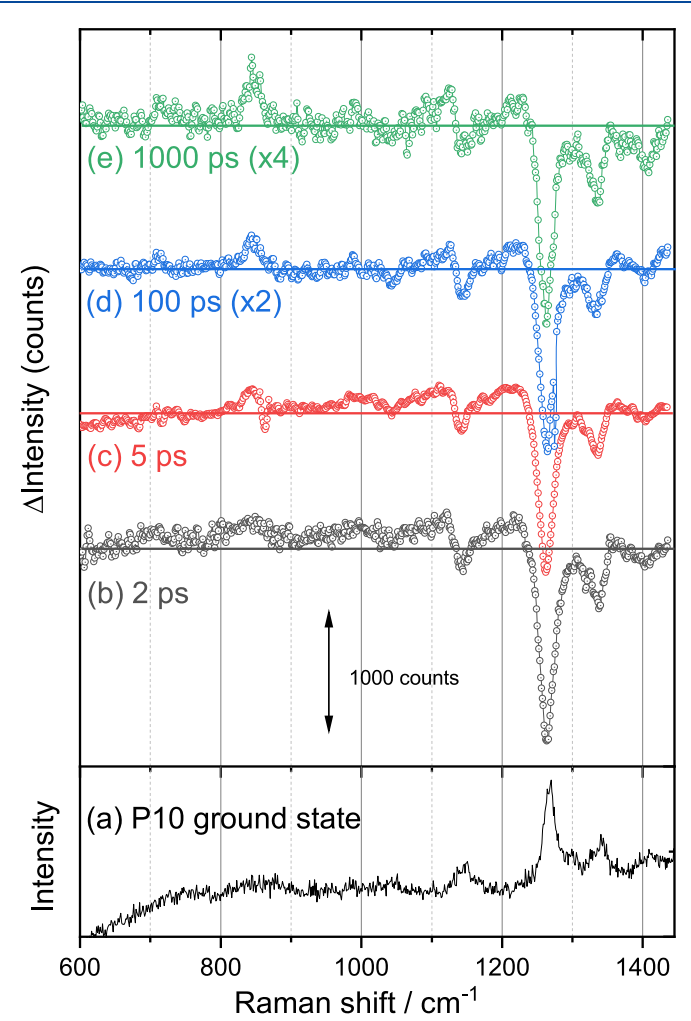

Figure 1. (a) Ground state Raman spectrum (633 nm probe) of P10 powder. $(\mathrm{b}-\mathrm{e}) \mathrm{TR}^{3}$ spectra of $\mathrm{P} 10$ recorded at the time indicated after $400 \mathrm{~nm}$ excitation of $\mathrm{P} 10$ powder by using a $630 \mathrm{~nm}$ Raman probe.

our TA experiment. In agreement with past reports of P10 in polar solvents, ${ }^{12}$ the TA spectra of the aggregates recorded following $400 \mathrm{~nm}$ excitation show a weak transient band between 620 and $660 \mathrm{~nm}$, assignable to either a P10 polaron pair or the $\mathrm{P} 10\left(\mathrm{e}^{-}\right)$polaron. This assignment is also supported by the species associated spectra generated through target 

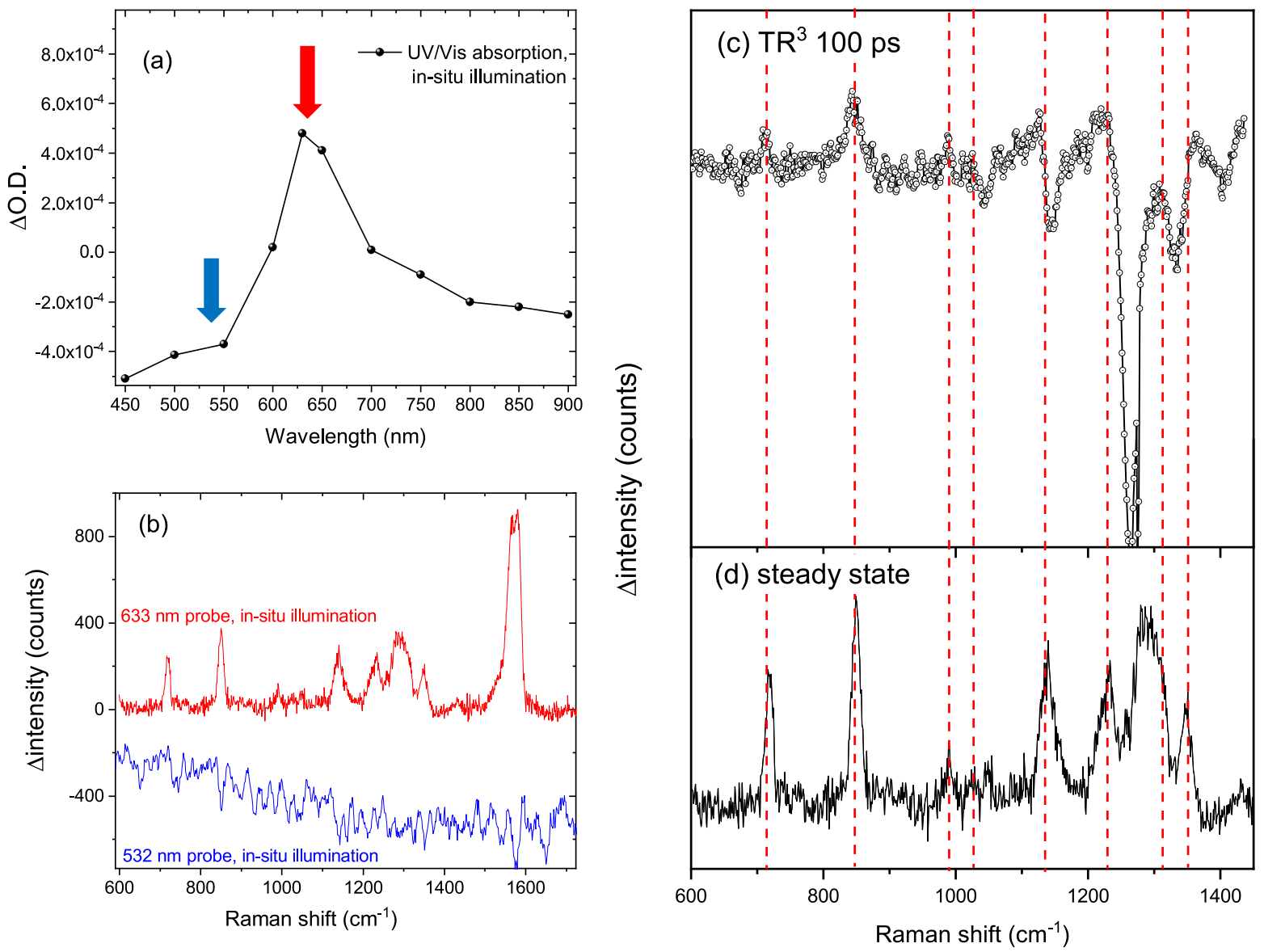

Figure 2. (a) UV-vis spectrum of P10 $\left(\mathrm{e}^{-}\right)$generated by CW $365 \mathrm{~nm}$ illumination of $\mathrm{P} 10$ in the presence of TEA/methanol/water (1:1:1). The red and blue arrows indicate the Raman probe wavelengths used in (b). (b) Raman difference spectrum (using the sample in the dark as a background) of P10 in the presence of TEA/methanol/water (1:1:1) on and off resonance with the observed feature in the UV-vis spectrum. (c, d) Comparison between the steady state (d) and $\mathrm{TR}^{3}$ Raman data (c, $100 \mathrm{ps}$ ) which show the presence of the same species.

analysis fitting of the TA data, based on the kinetic model derived within this Letter as a result of the $\mathrm{TR}^{3}$ data, Figure S5, and the accompanying text. The $\mathrm{TR}^{3}$ spectra of $\mathrm{P} 10$ powder following $400 \mathrm{~nm}$ excitation recorded by using a $630 \mathrm{~nm}$ Raman probe that is resonant with the proposed $\mathrm{P} 10$ polaron are shown in Figure $1 \mathrm{~b}-\mathrm{e}$. In common with many organic photocatalysts $\mathrm{P} 10$ is photoluminescent following excitation at energies greater than the optical gap $(2.61 \mathrm{eV}, \lambda<475 \mathrm{~nm}$; see Figure S3). ${ }^{12}$ Here we make use of an optically pumped Kerr gate to remove the majority of the photoluminescence (PL) background that otherwise masks the weak Raman scatter from the photogenerated transients (Scheme 1). ${ }^{28-30} 2$ ps after excitation of $\mathrm{P} 10$ the $\mathrm{TR}^{3}$ spectrum shows bleaching (a decrease in scattering intensity) of the ground state Raman modes of P10, and only broad excited state Raman bands are present, which are assigned to vibrationally hot photogenerated state(s) (Figure $1 \mathrm{~b}$ ). Within 5 ps these begin to cool, and transient Raman bands are observed. These are centered at 713,847 , and $988 \mathrm{~cm}^{-1}$ (weak) and in the region of 1210 and $1110 \mathrm{~cm}^{-1}$ (partially overlapped with the ground state bleaches). The new transient features persist for longer than 1 ns (Figure 1e).

Two experiments were performed to test the assignment of the transient Raman bands to either $\mathrm{P} 10\left(\mathrm{e}^{-}\right)$or the polaron pair. First, we recorded $\mathrm{TR}^{3}$ spectra in the presence of a sacrificial electron donor (TEA/methanol/water, 1:1:1) that will increase the yield and lifetime of the $\mathrm{P} 10\left(\mathrm{e}^{-}\right)$polaron. For a discussion of the implications of $\mathrm{P} 10\left(\mathrm{e}^{-}\right)$accumulation on the $\mathrm{TR}^{3}$ experiment, please refer to the text accompanying Figure S6. The $\mathrm{TR}^{3}$ spectra show transient bands at 719,849 , 1138,1262 , and $1331 \mathrm{~cm}^{-1}$, in good agreement with the $\mathrm{TR}^{3}$ data recorded in the absence of the sacrificial electron donor (Figure 1 and Figure S6). The $\mathrm{TR}^{3}$ bands at 1262 and 1331 $\mathrm{cm}^{-1}$ were not visible in the absence of the electron donor (Figure 1) due to the overlap with the P10 ground state bleach. Second, as $\mathrm{P} 10\left(\mathrm{e}^{-}\right)$accumulates under steady state illumination, ${ }^{23}$ we have also recorded the Raman spectra under $365 \mathrm{~nm}$ LED illumination using a conventional (steady state) microscope both on resonance $(633 \mathrm{~nm})$ and off resonance (532 $\mathrm{nm})$ with the known UV-vis absorption maximum of P10 $\left(\mathrm{e}^{-}\right)$ (Figure 2a, b). The Raman spectrum of the photogenerated $\mathrm{P} 10\left(\mathrm{e}^{-}\right)$by using a $633 \mathrm{~nm}$ probe wavelength shows excellent agreement with the proposed $\mathrm{P} 10\left(\mathrm{e}^{-}\right) \mathrm{TR}^{3}$ spectrum (Figure $2 \mathrm{c}$ ), while the spectrum recorded with a $532 \mathrm{~nm}$ probe under identical conditions shows no bands that can be assigned to a photogenerated species.

Preresonance $^{31}$ and resonance ${ }^{32}$ Raman spectra for P10, $\mathrm{P} 10\left(\mathrm{e}^{-}\right)$, and one-electron-oxidized $\mathrm{P} 10\left(\mathrm{P} 10\left(\mathrm{~h}^{+}\right)\right)$of the monomer and oligomers of different length have been predicted by $\mathrm{DFT}$ using the $\omega \mathrm{B} 97 \mathrm{XD}$ exchange-correlation functional $^{33}$ and the cc-pVDZ basis set. ${ }^{34,35}$ See Figure 3 for a comparison between the experimental spectra and predicted (pre)resonance spectra for a P10 hexamer and Figures S7-S10 for all predicted (pre)resonance spectra for the different 


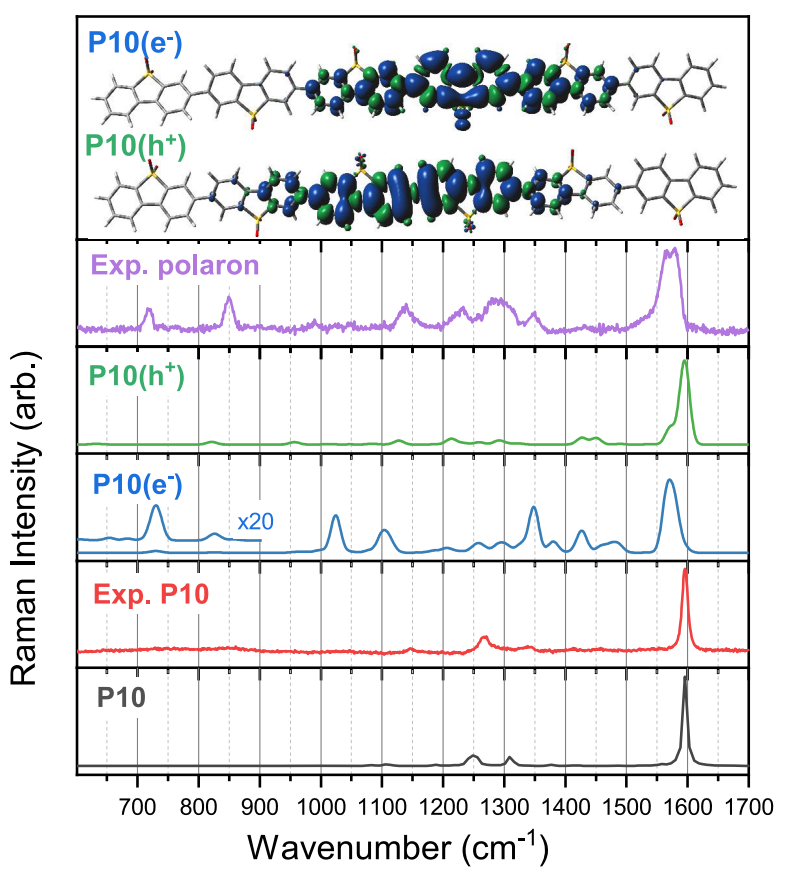

Figure 3. Comparison of the experimental spectra of $\mathrm{P} 10$ and the $\mathrm{P} 10$ polaron to the predicted (pre)resonance $(\omega \mathrm{B} 97 \mathrm{XD} / \mathrm{cc}-\mathrm{pVDZ})$ spectra of a P10 hexamer in different charge states. All predicted spectra have been scaled by a factor of 0.943 , obtained by aligning the intense peak in the predicted neutral spectrum to that of the P10 experimental spectrum. The predicted resonance Raman spectra shown for $\mathrm{P} 10\left(\mathrm{e}^{-}\right)$and $\mathrm{P} 10\left(\mathrm{~h}^{+}\right)$are calculated for the intermediate excited state with a predicted vertical absorption closest to $630 \mathrm{~nm}$. Similar spectra for other intermediate excited states can be found in Figure S10. The inset is a schematic of the polaron localization for the two charged species.

oligomer lengths and those predicted using another functional. All DFT predicted spectra discussed herein and within the Supporting Information have been scaled by the same factor, obtained by aligning the intense predicted P10 peak to that of experiment. For all oligomer lengths the predicted P10 preresonance Raman spectrum is dominated by a single intense transition with good agreement to its experimental counterpart (see Figure 3). The predicted resonance Raman spectra for P10 $\left(\mathrm{e}^{-}\right)$and $\mathrm{P} 10\left(\mathrm{~h}^{+}\right)$show an increased number of intense peaks below $1600 \mathrm{~cm}^{-1}$ which differ depending on the excited state the probe wavelength is on resonance with, complicating our ability to distinguish between the two species. However, for oligomers it is apparent that the calculations in the case of $\mathrm{P} 10\left(\mathrm{e}^{-}\right)$consistently reproduce the experimentally observed red-shift of the strong $1596 \mathrm{~cm}^{-1}$ peak of the P10 polaron species. This is not the case for its $\mathrm{P} 10\left(\mathrm{~h}^{+}\right)$ counterparts for which the intense peak is predicted to be unshifted relative to that of P10. Resonance Raman spectra predicted with a different range-separated exchange-correlation functional, CAM-B3LYP (see Figure S9) suggest that this redshift of the most intense peak in the Raman spectra is representative of $\mathrm{P} 10\left(\mathrm{e}^{-}\right)$oligomers and the lack of such a shift typical of their $\mathrm{P} 10\left(\mathrm{~h}^{+}\right)$counterparts. In Figure 3, the unpaired electron density is shown for the $\mathrm{P} 10\left(\mathrm{e}^{-}\right)$and $\mathrm{P} 10\left(\mathrm{~h}^{+}\right)$ hexamer species. For both species, the polaron is localized on the central P10 moieties, with the calculations of P10 $\left(\mathrm{e}^{-}\right)$ showing increased electron density on the thiophene ring.

These results indicate that by using the $\mathrm{TR}^{3}$ experiment we are measuring the spectrum of either the $\mathrm{P} 10\left(\mathrm{e}^{-}\right)$polaron or the vibrational modes associated with electron localization within a polaron pair. We now turn to the rate and mechanism of polaron formation in the absence and presence of a sacrificial electron donor (Figure 4). An advantage of the $\mathrm{TR}^{3}$

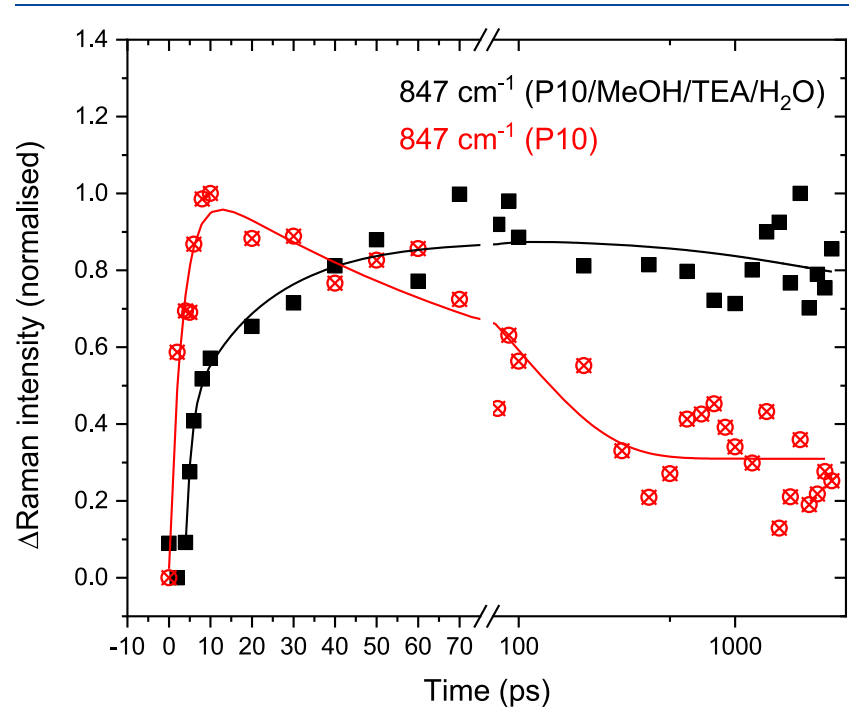

Figure 4. (a) Kinetics of P10 polaron Raman band at $847 \mathrm{~cm}^{-1}$ following $400 \mathrm{~nm}$ excitation. P10 powder (no electron donor) data are shown with red circles, and data with a $\mathrm{MeOH} / \mathrm{TEA} / \mathrm{H}_{2} \mathrm{O}$ sacrificial electron donor are shown with black squares. The parameters of the fit lines are described in the text and Table S5. The data are normalized (to 0,1 ) to allow easy comparison to the Raman kinetics of P10 in the absence of the sacrificial electron donor. The kinetics of the $847 \mathrm{~cm}^{-1}$ bands are baseline corrected by taking the difference in Raman scatter at 847 and $928 \mathrm{~cm}^{-1}$, where no transient bands are present.

experiment is that contributions from other off-resonance intermediates are minimal, simplifying the analysis of the transient data. The kinetics of the $847 \mathrm{~cm}^{-1}$ Raman band are studied, but all $\mathrm{TR}^{3}$ bands in this spectral region show similar kinetics. Following excitation of P10 in the absence of a sacrificial electron donor the decay can be well fitted to a biexponential function with an initial rise in intensity, which is close to the instrument response function $(\tau \sim 3 \mathrm{ps})$, and a subsequent slower decay (101 ps) to form a population that persists until the longest time scales studied (3.4 ns); full fitting parameters are in Table S5. The slow decay is not due to electron transfer from the P10 to residual Pd left during polymer synthesis, as this is known to occur on the microsecond and slower time scale; ${ }^{23}$ instead, it is assigned to recombination of the polaron pair. The fast rise in intensity of the $847 \mathrm{~cm}^{-1}$ band is in line with the observed rate of vibrational cooling (Figure S11), from which we estimate that the transient species reach thermal equilibrium by $\sim 10$ ps. After 10 ps, we see no further increase in intensity of the 847 $\mathrm{cm}^{-1}$ Raman mode (Figure 4). To probe the lifetime of the excitonic state, PL between 634 and $697 \mathrm{~nm}$ is measured by blocking the Raman probe beam during the Kerr gated experiment (Figure S12). The PL at $657 \mathrm{~nm}$ has an amplitudeweighted average lifetime of 314 ps (Table S6), demonstrating that the excitonic state of P10 persists at time scales beyond those where we observe polaron formation. This leads to the conclusion that polaron formation does not occur at significant levels from the thermalized exciton state, despite this species persisting for several hundred picoseconds. Instead, the 
thermalized exciton decays to the ground state, resulting in the slow recovery of the negative $\mathrm{P} 10$ band in the $\mathrm{TR}^{3}$ spectrum at $1265 \mathrm{~cm}^{-1}$ (Figure S13). In the time-resolved experiment P10 is excited with photon energies ca. $0.5 \mathrm{eV}$ greater than the P10 optical gap; ${ }^{12}$ this excess energy is important in enabling $\mathrm{P} 10\left(\mathrm{e}^{-}\right)$/polaron pair formation, with hot exciton dissociation being the dominant pathway for P10 in the absence of a sacrificial electron donor. Target analysis of the TA data (Figure S5 and accompanying text) shows that the TA data can be well fitted to the $\mathrm{TR}^{3}$-derived model with $\sim 95 \%$ of the polaron-pair population being generated with a lifetime of $\sim 4.5$ ps directly from the hot excitonic state.

In the presence of the sacrificial electron donor the P10 $\left(\mathrm{e}^{-}\right)$ Raman band ( $847 \mathrm{~cm}^{-1}$, Figure 4$)$ shows both a fast initial rise $(\tau<2 \mathrm{ps})$ and a second slower growth $(\tau \sim 20 \mathrm{ps})$. The similar fast lifetime component in the presence and absence of an electron donor shows that polaron formation occurs both directly from the hot excitonic state $(<2 \mathrm{ps})$ that is present for up to 10 ps (Figure S11) and via reductive quenching of the thermalized exciton by the amine electron donor ( $\sim 20 \mathrm{ps})$, with both processes contributing similar amounts to the overall amplitude (Table S5). We see only minimal decay of the 847 $\mathrm{cm}^{-1}$ band in the presence of the sacrificial electron donor, suggesting that hole transfer is occurring following the fast polaron-pair formation. The conclusion that reductive quenching of the relaxed excitonic state can occur is supported by PL measurements at $657 \mathrm{~nm}$ which show a decreased amplitude weighted lifetime (67 ps) with a fast decay component $(\tau \sim 13 \mathrm{ps})$ in the presence of the electron donor mix (Table S6 and Figure S13). A significant PL population persists to $>100 \mathrm{ps}$, showing that a proportion of the exciton population is inaccessible to the scavenger. Past studies have correlated the yield of $\mathrm{P} 10\left(\mathrm{e}^{-}\right)$to the driving force for hole transfer from the polymer exciton to a sacrificial electron donor, ${ }^{12}$ and we confirm the presence of this pathway. However, the observation that a similar fast rise in the 847 $\mathrm{cm}^{-1}$ band is existent in both the presence and absence of the electron donor suggests that fast exciton dissociation to form polaron pairs with subsequent electron transfer from the sacrificial electron donor is also occurring, and this is a major contributing factor to the high level of measured photocatalytic activity of P10 for hydrogen evolution.

More widely, hot exciton dissociation is expected to be of particular importance for other particulate polymer photocatalysts which exist as aggregates ranging from several hundred nanometers to micrometers. ${ }^{12}$ Most photons will be absorbed away from the polymer/solvent (water) interface. In addition to preventing access to the sacrificial electron donor, the absence of the high-dielectric environment presents a large barrier to dissociation, with past calculations ${ }^{36}$ of binding energies of $\sim 1.2 \mathrm{eV}$ for excitons within the polymer matrix of a similar linear polymer, as compared to only $\sim 0.17 \mathrm{eV}$ near the polymer/water interface. In the absence of charge separation occurring prior to thermalization driven by the excess energy of the hot exciton, most excitons formed away from the polymer/ interface would be lost via parasitical de-excitation.

In conclusion, we have shown that Kerr gated $\mathrm{TR}^{3}$ spectroscopy enables the study of ultrafast polaron electron formation in P10, a highly active hydrogen evolution photocatalyst under sacrificial conditions. More widely, we propose that it is a valuable technique for the study of photogenerated transients of polymer photocatalysts and photoelectrodes and could contribute to the effective design, for example, of Z-scheme composite materials for overall water splitting. A combination of experimental Raman spectroscopy and DFT calculations supports the assignment of the $\mathrm{TR}^{3}$ spectra, and the calculations indicate that the electron is predominantly localized on a single P10 moiety, in particular around the thiophene ring, which is beneficial given the proposed role of the sulfone groups in enabling water molecules to localize providing a more polar environment. ${ }^{12}$ Past models have focused on P10 $\left(\mathrm{e}^{-}\right)$formation through hole transfer from the excitonic state to the sacrificial amine. ${ }^{12}$ Here we also show that hole transfer following polaron-pair formation from hot states is also an important pathway for forming long-lived $\mathrm{P} 10\left(\mathrm{e}^{-}\right)$. It is known from OPV research that the distribution of excess energy following singlet exciton formation can have a critical role in facilitating charge separation, ${ }^{18}$ and our work also demonstrates the importance of polaron pair formation prior to thermalization for this polymer photocatalyst, P10.

\section{ASSOCIATED CONTENT}

\section{Supporting Information}

The Supporting Information is available free of charge at https://pubs.acs.org/doi/10.1021/acs.jpclett.1c03073.

Experimental procedures, details of the DFT calculations of P10 with different chain lengths, transient absorption data and additional $\mathrm{TR}^{3}$ data (PDF)

DFT optimized geometries of all relevant structures (ZIP)

\section{AUTHOR INFORMATION}

\section{Corresponding Authors}

Martijn A. Zwijnenburg - Department of Chemistry, University College London, London WC1H OAJ, U.K.; ○ orcid.org/0000-0001-5291-2130;

Email: m.zwijnenburg@ucl.ac.uk

Alexander J. Cowan - Stephenson Institute for Renewable Energy and Department of Chemistry, University of Liverpool, Liverpool L69 7ZF, U.K.; (1) orcid.org/00000001-9032-3548; Email: acowan@liverpool.ac.uk

\section{Authors}

Verity L. Piercy - Stephenson Institute for Renewable Energy and Department of Chemistry, University of Liverpool, Liverpool L69 7ZF, U.K.

Khezar H. Saeed - Stephenson Institute for Renewable Energy and Department of Chemistry, University of Liverpool, Liverpool L69 7ZF, U.K.

Andrew W. Prentice - Department of Chemistry, University College London, London WC1H OAJ, U.K.

Gaia Neri - Stephenson Institute for Renewable Energy and Department of Chemistry, University of Liverpool, Liverpool L69 7ZF, U.K.

Chao Li - Stephenson Institute for Renewable Energy and Department of Chemistry, University of Liverpool, Liverpool L69 7ZF, U.K.; Department of Chemistry and Materials Innovation Factory, University of Liverpool, Liverpool L7 $3 N Y$, U.K.

Adrian M. Gardner - Stephenson Institute for Renewable Energy and Department of Chemistry, University of Liverpool, Liverpool L69 7ZF, U.K. 
Yang Bai - Department of Chemistry and Materials Innovation Factory, University of Liverpool, Liverpool L7 3NY, U.K.

Reiner Sebastian Sprick - Department of Chemistry and Materials Innovation Factory, University of Liverpool, Liverpool L7 3NY, U.K.; Department of Pure and Applied Chemistry, University of Strathclyde, Glasgow G1 1XL, U.K.; 1 orcid.org/0000-0002-5389-2706

Igor V. Sazanovich - Central Laser Facility, Research Complex at Harwell, STFC Rutherford Appleton Laboratory, Didcot, Oxfordshire OX11 OQX, U.K.

Andrew I. Cooper - Department of Chemistry and Materials Innovation Factory, University of Liverpool, Liverpool L7 3NY, U.K.; ○ orcid.org/0000-0003-0201-1021

Matthew J. Rosseinsky - Department of Chemistry and Materials Innovation Factory, University of Liverpool, Liverpool L7 3NY, U.K.; (1) orcid.org/0000-0002-19102483

Complete contact information is available at: https://pubs.acs.org/10.1021/acs.jpclett.1c03073

\section{Author Contributions}

V.L.P., K.H.S., and A.W.P. contributed equally to this work.

\section{Notes}

The authors declare no competing financial interest.

\section{ACKNOWLEDGMENTS}

The $\mathrm{TR}^{3}$ experiments were performed at the UK Central Laser Facility using the ULTRA apparatus as part of experiment 19230022. Transient absorption spectroscopic measurements were performed at the University of Liverpool ECR Laser Laboratory supported by UKRI-EPSRC grant EP/S017623/1 and the University of Liverpool. Prof L. Hardwick is thanked for access to the Raman steady-state spectrometer. Funding from UKRI-EPSRC (grants EP/N010531/1 and EP/ P034497/1) and the Leverhulme Trust (grant RPG-2019209) is also gratefully acknowledged. R.S.S. thanks the University of Strathclyde for financial support through The Strathclyde Chancellor's Fellowship Scheme.

\section{REFERENCES}

(1) Wang, Y.; Vogel, A.; Sachs, M.; Sprick, R. S.; Wilbraham, L.; Moniz, S. J. A.; Godin, R.; Zwijnenburg, M. A.; Durrant, J. R.; Cooper, A. I.; Tang, J. Current Understanding and Challenges of Solar-Driven Hydrogen Generation Using Polymeric Photocatalysts. Nature Energy 2019, 4, 746-760.

(2) Wang, X.; Maeda, K.; Thomas, A.; Takanabe, K.; Xin, G.; Carlsson, J. M.; Domen, K.; Antonietti, M. A Metal-Free Polymeric Photocatalyst for Hydrogen Production from Water under Visible Light. Nat. Mater. 2009, 8 (1), 76-80.

(3) Sprick, R. S.; Jiang, J.-X.; Bonillo, B.; Ren, S.; Ratvijitvech, T.; Guiglion, P.; Zwijnenburg, M. A.; Adams, D. J.; Cooper, A. I. Tunable Organic Photocatalysts for Visible-Light-Driven Hydrogen Evolution. J. Am. Chem. Soc. 2015, 137 (9), 3265-3270.

(4) Meier, C. B.; Clowes, R.; Berardo, E.; Jelfs, K. E.; Zwijnenburg, M. A.; Sprick, R. S.; Cooper, A. I. Structurally Diverse Covalent Triazine-Based Framework Materials for Photocatalytic Hydrogen Evolution from Water. Chem. Mater. 2019, 31 (21), 8830-8838.

(5) Lan, Z.-A.; Fang, Y.; Zhang, Y.; Wang, X. Photocatalytic Oxygen Evolution from Functional Triazine-Based Polymers with Tunable Band Structures. Angew. Chem., Int. Ed. 2018, 57 (2), 470-474.

(6) Bi, J.; Fang, W.; Li, L.; Wang, J.; Liang, S.; He, Y.; Liu, M.; Wu, L. Covalent Triazine-Based Frameworks as Visible Light Photo- catalysts for the Splitting of Water. Macromol. Rapid Commun. 2015, 36 (20), 1799-1805.

(7) Vyas, V. S.; Haase, F.; Stegbauer, L.; Savasci, G.; Podjaski, F.; Ochsenfeld, C.; Lotsch, B. V. A Tunable Azine Covalent Organic Framework Platform for Visible Light-Induced Hydrogen Generation. Nat. Commun. 2015, 6 (1), 8508.

(8) Wang, X.; Chen, L.; Chong, S. Y.; Little, M. A.; Wu, Y.; Zhu, W.H.; Clowes, R.; Yan, Y.; Zwijnenburg, M. A.; Sprick, R. S.; Cooper, A. I. Sulfone-Containing Covalent Organic Frameworks for Photocatalytic Hydrogen Evolution from Water. Nat. Chem. 2018, 10 (12), 1180-1189.

(9) Pachfule, P.; Acharjya, A.; Roeser, J.; Langenhahn, T.; Schwarze, M.; Schomäcker, R.; Thomas, A.; Schmidt, J. Diacetylene Functionalized Covalent Organic Framework (COF) for Photocatalytic Hydrogen Generation. J. Am. Chem. Soc. 2018, 140, 1423-1427.

(10) Pati, P. B.; Damas, G.; Tian, L.; Fernandes, D. L. A.; Zhang, L.; Pehlivan, I. B.; Edvinsson, T.; Araujo, C. M.; Tian, H. An Experimental and Theoretical Study of an Efficient Polymer NanoPhotocatalyst for Hydrogen Evolution. Energy Environ. Sci. 2017, 10 (6), $1372-1376$.

(11) Sprick, R. S.; Bonillo, B.; Clowes, R.; Guiglion, P.; Brownbill, N. J.; Slater, B. J.; Blanc, F.; Zwijnenburg, M. A.; Adams, D. J.; Cooper, A. I. Visible-Light-Driven Hydrogen Evolution Using Planarized Conjugated Polymer Photocatalysts. Angew. Chem., Int. Ed. 2016, 55 (5), 1792-1796.

(12) Sachs, M.; Sprick, R. S.; Pearce, D.; Hillman, S. A. J.; Monti, A.; Guilbert, A. A. Y.; Brownbill, N. J.; Dimitrov, S.; Shi, X.; Blanc, F.; Zwijnenburg, M. A.; Nelson, J.; Durrant, J. R.; Cooper, A. I. Understanding Structure-Activity Relationships in Linear Polymer Photocatalysts for Hydrogen Evolution. Nat. Commun. 2018, 9 (1), 4968.

(13) Bai, Y.; Nakagawa, K.; Cowan, A. J.; Aitchison, C. M.; Yamaguchi, Y.; Zwijnenburg, M. A.; Kudo, A.; Sprick, R. S.; Cooper, A. I. Photocatalyst Z-Scheme System Composed of a Linear Conjugated Polymer and BiVO 4 for Overall Water Splitting under Visible Light. J. Mater. Chem. A 2020, 8, 16283.

(14) Sprick, R. S.; Chen, Z.; Cowan, A. J.; Bai, Y.; Aitchison, C. M.; Fang, Y.; Zwijnenburg, M. A.; Cooper, A. I.; Wang, X. Water Oxidation with Cobalt-Loaded Linear Conjugated Polymer Photocatalysts. Angew. Chem., Int. Ed. 2020, 59 (42), 18695-18700.

(15) Fu, Z.; Vogel, A.; Zwijnenburg, M. A.; Cooper, A. I.; Sprick, R. S. Photocatalytic Syngas Production Using Conjugated Organic Polymers. J. Mater. Chem. A 2021, 9 (7), 4291-4296.

(16) De Sio, A.; Troiani, F.; Maiuri, M.; Rehault, J.; Sommer, E.; Lim, J.; Huelga, S. F.; Plenio, M. B.; Rozzi, C. A.; Cerullo, G.; Molinari, E.; Lienau, C. Tracking the Coherent Generation of Polaron Pairs in Conjugated Polymers. Nat. Commun. 2016, 7 (1), 1-8.

(17) Tautz, R.; Da Como, E.; Limmer, T.; Feldmann, J.; Egelhaaf, H.-J.; von Hauff, E.; Lemaur, V.; Beljonne, D.; Yilmaz, S.; Dumsch, I.; Allard, S.; Scherf, U. Structural Correlations in the Generation of Polaron Pairs in Low-Bandgap Polymers for Photovoltaics. Nat. Commun. 2012, 3 (1), 1-8.

(18) Pensack, R. D.; Asbury, J. B. Beyond the Adiabatic Limit: Charge Photogeneration in Organic Photovoltaic Materials. J. Phys. Chem. Lett. 2010, 1 (15), 2255-2263.

(19) Kaake, L. G.; Jasieniak, J. J.; Bakus, R. C.; Welch, G. C.; Moses, D.; Bazan, G. C.; Heeger, A. J. Photoinduced Charge Generation in a Molecular Bulk Heterojunction Material. J. Am. Chem. Soc. 2012, 134 (48), 19828-19838.

(20) Miao, T. J.; Tang, J. Characterization of Charge Carrier Behavior in Photocatalysis Using Transient Absorption Spectroscopy. J. Chem. Phys. 2020, 152 (19), 194201.

(21) Forster, M.; Cheung, D. W. F.; Gardner, A. M.; Cowan, A. J. Potential and Pitfalls: On the Use of Transient Absorption Spectroscopy for in Situ and Operando Studies of Photoelectrodes. J. Chem. Phys. 2020, 153, 150901.

(22) Kosco, J.; Sachs, M.; Godin, R.; Kirkus, M.; Francas, L.; Bidwell, M.; Qureshi, M.; Anjum, D.; Durrant, J. R.; McCulloch, I. The Effect of Residual Palladium Catalyst Contamination on the 
Photocatalytic Hydrogen Evolution Activity of Conjugated Polymers. Adv. Energy Mater. 2018, 8 (34), 1802181.

(23) Sachs, M.; Cha, H.; Kosco, J.; Aitchison, C. M.; Francàs, L.; Corby, S.; Chiang, C.-L.; Wilson, A.; Godin, R.; Fahey-Williams, A.; Cooper, A.; Sprick, S.; McCulloch, I.; Durrant, J. R. Tracking Charge Transfer to Residual Metal Clusters in Conjugated Polymers for Photocatalytic Hydrogen Evolution. J. Am. Chem. Soc. 2020, 142, 14574.

(24) Magnanelli, T. J.; Bragg, A. E. Time-Resolved Raman Spectroscopy of Polaron Pair Formation in Poly(3-Hexylthiophene) Aggregates. J. Phys. Chem. Lett. 2015, 6, 438.

(25) Takaya, T.; Enokida, I.; Furukawa, Y.; Iwata, K. Direct Observation of Structure and Dynamics of Photogenerated Charge Carriers in Poly(3-Hexylthiophene) Films by Femtosecond TimeResolved Near-IR Inverse Raman Spectroscopy. Molecules 2019, 24 (3), 431

(26) Yu, W.; Zhou, J.; Bragg, A. E. Exciton Conformational Dynamics of Poly(3-Hexylthiophene) (P3HT) in Solution from Time-Resolved Resonant-Raman Spectroscopy. J. Phys. Chem. Lett. 2012, 3 (10), 1321-1328.

(27) Yu, W.; Donohoo-Vallett, P. J.; Zhou, J.; Bragg, A. E. Ultrafast Photo-Induced Nuclear Relaxation of a Conformationally Disordered Conjugated Polymer Probed with Transient Absorption and Femtosecond Stimulated Raman Spectroscopies. J. Chem. Phys. 2014, 141 (4), 044201.

(28) Benniston, A. C.; Matousek, P.; McCulloch, I. E.; Parker, A. W.; Towrie, M. Detailed Picosecond Kerr-Gated Time-Resolved Resonance Raman Spectroscopy and Time-Resolved Emission Studies of Merocyanine 540 in Various Solvents. J. Phys. Chem. A 2003, 107 (22), 4347-4353.

(29) Keshari, S.; And, S.; Umapathy, S.; Parker, A. W. Focal Point Review Time-Resolved Resonance Raman Spectroscopy: Exploring Reactive Intermediates. Appl. Spectrosc. 2011, 65 (10), 1087-1115.

(30) Stanley, A.; Parker, A. W.; Towrie, M.; Matousek, P. Efficient Rejection of Fluorescence from Raman Spectra Using Picosecond Kerr Gating. Appl. Spectrosc. 1999, 53 (12), 1485-1489.

(31) Cheeseman, J. R.; Frisch, M. J. Basis Set Dependence of Vibrational Raman and Raman Optical Activity Intensities. J. Chem. Theory Comput. 2011, 7 (10), 3323-3334.

(32) Baiardi, A.; Bloino, J.; Barone, V. A General Time-Dependent Route to Resonance-Raman Spectroscopy Including Franck-Condon, Herzberg-Teller and Duschinsky Effects. J. Chem. Phys. 2014, 141 (11), 114108.

(33) Chai, J.-D.; Head-Gordon, M. Long-Range Corrected Hybrid Density Functionals with Damped Atom-Atom Dispersion Corrections. Phys. Chem. Chem. Phys. 2008, 10 (44), 6615-6620.

(34) Woon, D. E.; Dunning, T. H., Jr. Gaussian Basis Sets for Use in Correlated Molecular Calculations. III. The Atoms Aluminum through Argon. J. Chem. Phys. 1993, 98 (2), 1358.

(35) Dunning, T. H. Gaussian Basis Sets for Use in Correlated Molecular Calculations. I. The Atoms Boron through Neon and Hydrogen. J. Chem. Phys. 1989, 90 (2), 1007.

(36) Guiglion, P.; Butchosa, C.; Zwijnenburg, M. A. Polymer Photocatalysts for Water Splitting: Insights from Computational Modeling. Macromol. Chem. Phys. 2016, 217 (3), 344-353. 